\title{
Detection of carious lesions utilizing depolarization imaging by polarization sensitive optical coherence tomography
}

Jonas Golde Florian Tetschke Julia Walther Tobias Rosenauer Franz Hempel Christian Hannig Edmund Koch Lars Kirsten 


\title{
Detection of carious lesions utilizing depolarization imaging by polarization sensitive optical coherence tomography
}

\author{
Jonas Golde, ${ }^{a, *, \dagger}$ Florian Tetschke, ${ }^{\mathrm{a}, \mathrm{b}, \dagger}$ Julia Walther, ${ }^{\mathrm{a}, \mathrm{c}}$ Tobias Rosenauer, ${ }^{\mathrm{b}}$ Franz Hempel, ${ }^{\mathrm{a}}$ Christian Hannig, \\ Edmund Koch, ${ }^{a}$ and Lars Kirsten ${ }^{a}$ \\ ${ }^{a}$ Technische Universität Dresden, Faculty of Medicine Carl Gustav Carus, Anesthesiology and Intensive Care Medicine, \\ Clinical Sensoring and Monitoring, Dresden, Germany \\ ${ }^{\mathrm{b} T e c h n i s c h e}$ Universität Dresden, Faculty of Medicine Carl Gustav Carus, Policlinic of Operative and Pediatric Dentistry, Dresden, Germany \\ ${ }^{\top}$ Technische Universität Dresden, Faculty of Medicine Carl Gustav Carus, Department of Medical Physics and Biomedical Engineering, \\ Dresden, Germany
}

\begin{abstract}
As dental caries is one of the most common diseases, the early and noninvasive detection of carious lesions plays an important role in public health care. Optical coherence tomography (OCT) with its ability of depth-resolved, high-resolution, noninvasive, fast imaging has been previously recognized as a promising tool in dentistry. Additionally, polarization sensitive imaging provides quantitative measures on the birefringent tissue properties and can be utilized for imaging dental tissue, especially enamel and dentin. By imaging three exemplary tooth samples ex vivo with proximal white spot, brown spot, and cavity, we show that the combination of polarization sensitive OCT and the degree of polarization uniformity (DOPU) algorithm is a promising approach for the detection of proximal carious lesions due to the depolarization contrast of demineralized tissue. Furthermore, we investigate different sizes of the DOPU evaluation kernel on the resulting contrast and conclude a suitable value for this application. We propose that DOPU provides an easy to interpret image representation and appropriate contrast for possible future screening applications in early caries diagnostics. () The Authors. Published by SPIE under a Creative Commons Attribution 3.0 Unported License. Distribution or reproduction of this work in whole or in part requires full attribution of the original publication, including its DOI. [DOI: 10.1117/1.JBO.23.7.071203]
\end{abstract}

Keywords: optical coherence tomography; dentistry; polarization contrast; dental caries; biomedical imaging; depolarization. Paper 170643SSR received Oct. 1, 2017; accepted for publication Dec. 22, 2017; published online Jan. 26, 2018; corrected Feb. 21, 2018.

\section{Introduction}

The early identification of proximal carious lesions is still one of the most challenging issues in modern cariology and odontology, whereas several visual, optical, and radiographic techniques have been developed and improved in recent years. ${ }^{1,2}$ Providing depthresolved, high-resolution images of biological samples, optical coherence tomography (OCT) is a promising tool for detecting caries noninvasively, since first investigations of dental hard tissue in vitro and in vivo were performed by $\mathrm{OCT},{ }^{3,4}$ and imaging in the near-infrared range proved convenient scattering and absorption properties. ${ }^{5}$ So far, OCT has shown the potential to provide effective and reproducible diagnoses for clinical applications, ${ }^{6}$ and algorithms that enable early caries segmentation from conventional OCT images have been presented. ${ }^{7}$ Additionally to the structural imaging based on backscattering intensities, miscellaneous modalities and extensions for OCT have been developed, which provide tissue-dependent contrast. This includes polarization sensitive imaging and the consequent measurement of depthresolved birefringence, which until now has been applied in many biomedical as well as nonmedical fields. ${ }^{8,9}$ In terms of hard dental tissue and caries detection, first polarization sensitive OCT (PS-OCT) measurements confirmed the birefringent properties of enamel and dentin, ${ }^{4,10}$ and it was determined that

*Address all correspondence to: Jonas Golde, E-mail: jonas.golde @tu-dresden de

TThese authors contributed equally to this work demineralization, accompanying carious infections, results in depolarization of light. ${ }^{11}$ Since then, several studies have shown the potential of PS-OCT to observe the internal structure and the remineralization of lesions, as well as an early detection of variously located caries. ${ }^{12-14}$ Thereby, the occurring depolarization effect for linearly polarized incident light is usually evaluated by an intensity measurement of the perpendicular polarization state. $^{15}$

Complementary to the development of PS-OCT imaging for dental hard tissue, the assessment of depolarizing properties as a tissue specific contrast in ophthalmology led to the definition of the degree of polarization uniformity (DOPU) as a tool for retinal pigment epithelium segmentation. ${ }^{16}$ Since OCT as an interferometric technique only measures the contribution of fully polarized light, following the Jones formalism, it is impossible to determine the degree of polarization (DOP) from a single speckle or measurement. The DOPU algorithm, therefore, averages the Stokes components over adjacent speckles, and the underlying polarization scrambling results in a decreased DOP in the evaluation window. Based on DOPU, several improvements concerning its noise sensitivity, orientation dependency, and evaluation kernel size have been proposed ${ }^{9,17-20}$ and enhance depolarization imaging as a powerful tool for several biomedical applications.

Therefore, we demonstrate that depolarization imaging based on the DOPU algorithm could be a convenient and significant method in cariology to differentiate early and advanced stages of carious lesions from sound dental tissue. Additionally, we exemplary evaluate the impact of different kernel sizes on the DOPU 
contrast in this case and show that depolarization imaging offers images, which are easy to interpret.

\section{Materials and Methods}

\subsection{Experimental Setup}

To perform polarization sensitive measurements, we set up a custom swept source OCT system (Fig. 1) with single-mode fibers and a bulk optics scanner head, which is mainly based on a concept initially proposed by Hee et al. ${ }^{21}$ A swept source laser (Axsun Technologies Inc., Billerica, Massachusetts) with a sweep rate of $50 \mathrm{kHz}$, a center wavelength of $\lambda_{0}=1310 \mathrm{~nm}$, and a spectral bandwidth of $\Delta \lambda=110 \mathrm{~nm}$ (used for image processing) is connected via fiber couplers (FC) to a fiber Bragg grating (FBG), to create a phase stable sweep trigger signal, and to a booster optical amplifier to compensate later coupling losses in the interferometer setup. Polarization controllers (PCs) allow the alignment of linearly polarized light incident into the polarization-dependent booster optical amplifier (BOA) and the bulk optics setup, which is connected via an optical circulator (OC). An initially passed polarizing beam splitter (PBS) operates in forward direction as a polarizer, before a nonpolarizing beam splitter (BS) equally separates the light into reference and sample arm. The polarization rotation of the reference light results in a diagonal polarization, as the quarter wave plate (QWP) in the reference arm has an orientation of $22.5 \mathrm{deg}$ with respect to the optical axis. Contrarily, the incident light on the sample is circularly polarized due to a 45-deg-oriented QWP in the sample arm. Therefore, the returning sample light obtains an arbitrary elliptical polarization state depending on the sample's birefringence. The superposed light from reference and sample arm is then split by two PBS into both orthogonal polarization states, which finally results via collimating optics and single-mode fibers in a dual balanced detection of the co- and cross-polarization interference spectra. To acquire the data linear in $k$-space, the clock signal of the swept laser is utilized as a sampling trigger of the high-speed digitizer (Alazar Technologies Inc., PointeClaire, Canada).

For this study, volumes of $1280 \times 1280$ A-scans with a step size of $8 \mu \mathrm{m}$ in both lateral directions and a total imaging depth of $4.96 \mathrm{~mm}$, corresponding to 1024 pixels or an axial pixel size of $4.8 \mu \mathrm{m}$ in air, were recorded. The lateral and the axial resolutions in air were measured to be 15.6 and $15.1 \mu \mathrm{m}$, respectively. All data acquisition and image processing were performed by customized LabVIEW (National Instruments Inc., Austin, Texas) and Fiji ${ }^{22}$ software.

\subsection{Image Calculation and Representation}

As the underlying concept of polarization, Jones and Stokes formalisms, and image formation in conventional as well as PS-OCT with circularly polarized incident light on the sample and the presented detection scheme is widely described and was recently summarized by de Boer et al., ${ }^{9}$ the image formation from the spectra will be outlined here in brief.

The inverse Fourier transform of the balanced and linearly in $k$-space detected interference spectra for both cross- and co-polarization channel results in complex, depth-resolved A-scans with amplitudes $A_{x}(z), A_{y}(z)$, and phases $\phi_{x}(z)$, $\phi_{y}(z)$, and phase differences $\Delta \varphi(z)=\varphi_{y}(z)-\varphi_{x}(z)$, respectively. Hence, the reflectivity $R(z)$, the retardation $\delta(z)$, and the fast axis orientation $\theta(z)$ can be calculated as

$$
\begin{aligned}
& R(z) \propto A_{x}(z)^{2}+A_{y}(z)^{2}, \\
& \delta(z)=\arctan \left(\frac{A_{y}(z)}{A_{x}(z)}\right), \\
& \theta_{s}(z)=\frac{\pi-\Delta \phi(z)}{2} .
\end{aligned}
$$

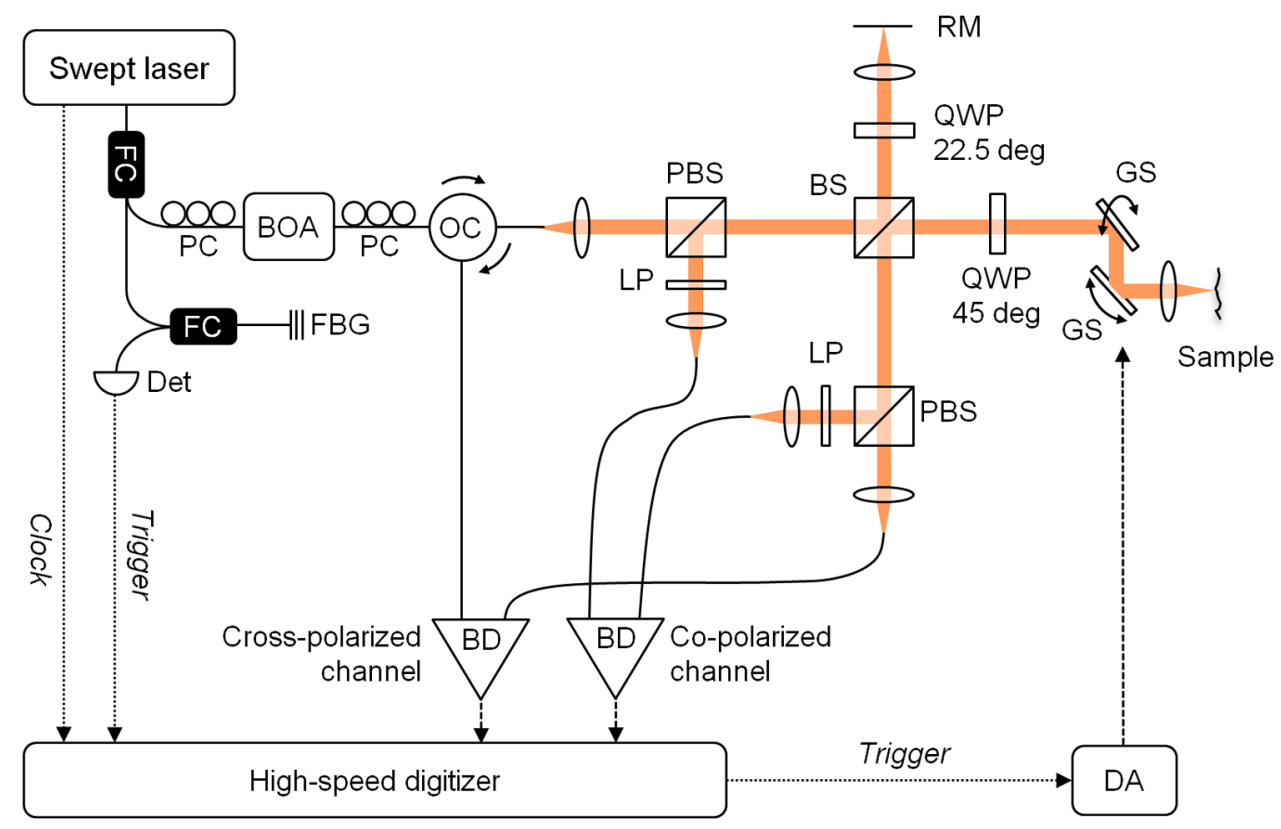

Fig. 1 PS-OCT system with swept laser $\left(\lambda_{0}=1310 \mathrm{~nm}, \Delta \lambda=110 \mathrm{~nm}\right)$. FC, fiber couplers, Det, detectors; FBG, fiber Bragg grating; PC, polarization controllers; BOA, booster optical amplifier; OC, optical circulator; PBS, polarization beam splitters; LP, linear polarizers; BS, polarization independent beam splitter; QWP, quarter wave plates oriented at 22.5 deg and 45 deg; RM, reference mirror; GS, galvanometer scanners; $\mathrm{BD}$, balanced detectors; and DA, data acquisition card. 
From the calculus' symmetry derive unambiguous measurement ranges of $[0 ; \pi / 2]$ for $\delta(z)$ and $[-\pi / 2 ; \pi / 2]$ for $\theta(z)$. For visualization, we chose a color gradient of blue, cyan, green, yellow, and red for $\delta(z)$ (usually known as "jet" scale) and a transition from purple over blue, white and red to purple for $\theta(z)$, as $-\pi / 2$ and $\pi / 2$ represent the same orientation.

Additionally, the Stokes vector elements $I, Q, U$, and $V$ can be calculated for each depth pixel

$$
S=\left(\begin{array}{c}
I \\
Q \\
U \\
V
\end{array}\right)=\left(\begin{array}{c}
A_{x}^{2}+A_{y}^{2} \\
A_{x}^{2}-A_{y}^{2} \\
2 A_{x} A_{y} \cos \Delta \phi \\
2 A_{x} A_{y} \sin \Delta \phi
\end{array}\right) \text {. }
$$

Applying the conventional degree of polarization calculus $\mathrm{DOP}=\left(Q_{\text {norm }}^{2}+U_{\text {norm }}^{2}+V_{\text {norm }}^{2}\right)^{1 / 2}$ pixel by pixel on the thus derived Stokes components would result in a DOP $=1$ (fully polarized) for the entire image, so that an averaging of $U, Q$, and $V$ for adjacent pixel in a certain evaluation kernel is introduced, ${ }^{16}$ which leads to the DOPU

$$
\mathrm{DOPU}=\sqrt{Q_{\text {norm,mean }}^{2}+U_{\text {norm,mean }}^{2}+V_{\text {norm,mean }}^{2}} .
$$

As the resulting DOPU is highly dependent on the number of speckles that are taken into account for averaging, and the speckle size itself depends on the axial and lateral resolutions, ${ }^{23}$ we decided to evaluate the kernel size as a multiple of axial and lateral resolution. Consequently, the chosen kernel size of $3 \times 3$ corresponds to an averaging of 9 (axial) $\times 6$ (lateral) pixels. To maintain image sizes and enable real-time processing, a recursive two-dimensional moving average algorithm, which avoids redundant calculation steps, was implemented. The chosen color transition for representing fully depolarized (0) up to completely polarized (1) light comprises red, yellow, and green, whereas yellow matches for a better contrast not the center (0.5) but a DOPU of about 0.7 in this dimensionless quantity.

Furthermore, a gray mask was applied on retardation, orientation, and DOPU images, which is based on a 16-dB threshold of the reflectivity data and overlays image regions with noisy or without sample information. Since the ratio between axial and lateral pixel sizes was not corrected for a superior depiction of depth information, all scale bars correspond to $500 \mu \mathrm{m}$, whereby axial depths are consistently adjusted on the refractive index of enamel in the 1300-nm range of $1.631 .^{24}$

\subsection{Tooth Samples}

Based on German regulations for this type of research, no ethical approval was mandatory for this study. ${ }^{25}$ Three extracted human molar teeth with proximal lesions were used for PS-OCT imaging. The teeth were provided by enretec $\mathrm{GmbH}$ (Velten, Germany) and were stored in a distilled water-thymol solution to prevent dehydration. The selected teeth were visually examined by an experienced dentist and showed the following characteristics:

- Tooth 1: Molar tooth with initial demineralization (white spot).

- Tooth 2: Molar tooth with discolored demineralization (brown spot).

- Tooth 3: Molar tooth with advanced carious lesion, penetrating to the dentin-enamel junction (DEJ) (cavity).

\section{Results and Discussion}

\subsection{Polarization Sensitive Imaging}

To demonstrate the capability of the PS-OCT system for measuring birefringence in dental hard tissue and likewise comparing the effect of an early carious lesion on the different representation modalities, Fig. 2 shows the intensities measured by co- and cross-polarization channels as well as the resulting reflectivity. Furthermore, retardation and optical axis orientation are shown next to the DOPU for a $3 \times 3$ (multiples of resolution) evaluation kernel according to Eqs. (1) and (3), respectively. The presented B-scan of the proximal side of an extracted human molar tooth corresponds to the same region as presented in Fig. 5 for "white

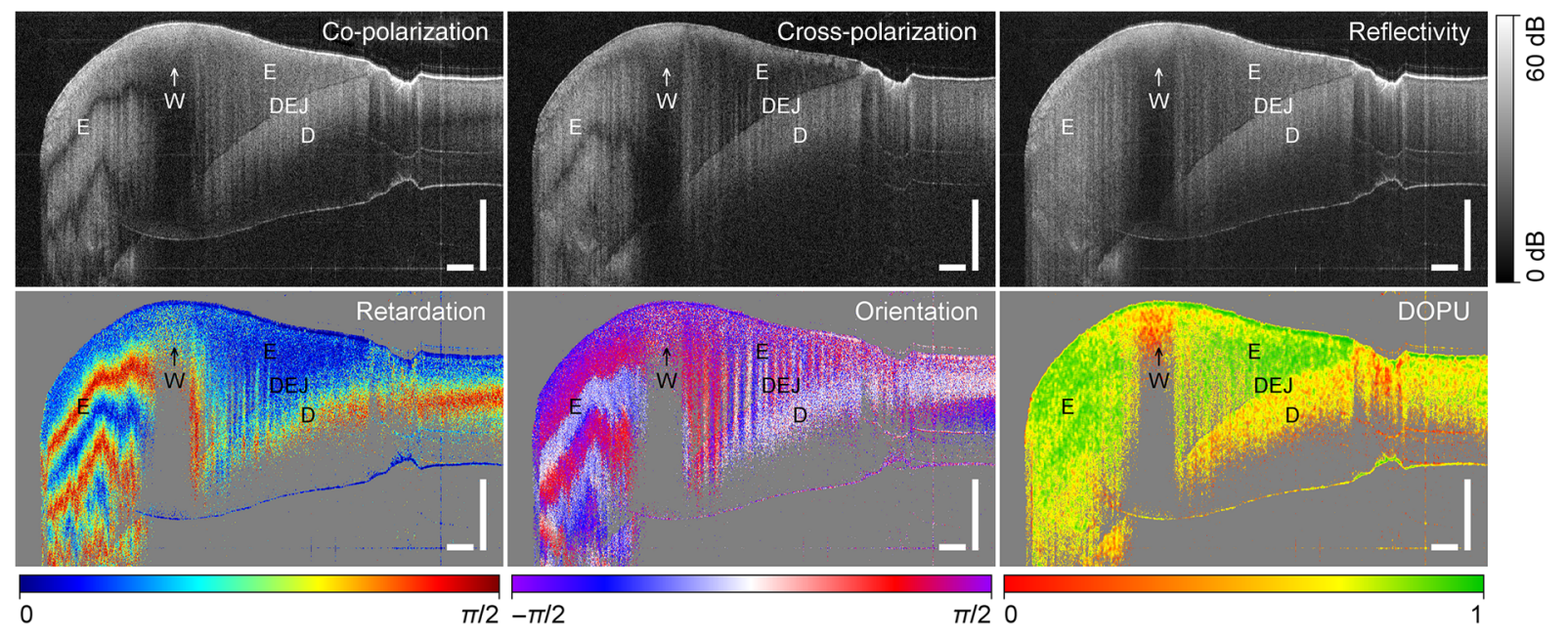

Fig. 2 OCT B-scan of the co-, cross-polarization channels and the determined reflectivity image showing enamel (E), the dentin-enamel junction (DEJ), dentin (D), and white spot (W) of an extracted human molar tooth. For polarization contrast imaging, the retardation, the fast axis orientation, and the DOPU $(3 \times 3$ kernel) are displayed. This B-scan is an adjacent of the B-scan as shown in Fig. 5 for "white spot." Scale bars correspond to $500 \mu \mathrm{m}$. 
spot." Enamel (E), dentin (D), and the dentin-enamel junction (DEJ) are labeled in the images as well as the region that has been identified as a white spot (W). In addition to the visible dental structures, imaging artifacts such as the mirrored surface reflex, which is especially discoverable in the lower half of the co-polarization image, appear due a coherence revival in the swept laser and should not be confused with actual sample structures.

As it can be clearly seen in the retardation as well as co- and cross-polarization images, the high birefringence of sound enamel becomes visible for an incident beam perpendicular to the enamel rods or prisms, which are oriented approximately perpendicular to the surface. ${ }^{26}$ While this is the case in the left third, the enamel rods in the center third are commonly parallel oriented to the incident beam and the retardation remains constant over the depth. Reversely, the orientation image allows an estimation of the rod structure in this center image region, but further investigations of the polarization sensitive image formation with regards to the precise spatial distribution ${ }^{27}$ are necessary for a conclusive interpretation. For dentin, less birefringence related to its substructure ${ }^{26}$ and a decreased DOPU, which has been previously observed ${ }^{28}$ and occurs due to multiple scattering, ${ }^{29}$ allow a delimitation from the surrounding enamel, as well as the in the intensity images visible DEJ.

Additionally, a white spot, an early stage of caries, and a case of demineralization in the outer enamel are present here. Due to a reduced penetration depth, a differentiation of the white spot from the nearby sound tissue is possible in this case for all modalities. However, while the comparison of co- and crosspolarization images with altered intensity ratios as well as the particular textures in the retardation and orientation images might enable a trained expert to recognize this feature, the
DOPU offers an unambiguous contrast for carious lesions and demineralization.

Moreover, a decreased DOPU can be observed in the right image third near the enamel-cementum intersection, which is assumed as an artificial deformation, possibly by the mechanical impact during the extraction of the molar tooth.

\subsection{DOPU Evaluation Kernel}

We compared different symmetric sizes of the evaluation kernel that is used for averaging the Stokes components $Q, U$, and $V$ [Eq. (2)] in a certain window before the DOPU is calculated [Eq. (3)]. As this refers to multiples of the axial and lateral resolution, a kernel size of $1 \times 1$ corresponds to $3 \times 2$ pixels (axial $\times$ lateral dimension) and a kernel size of $6 \times 6$ to $19 \times 12$ pixels. The resulting images in Fig. 3 show an increasing contrast with increasing kernel size, whereby the resolution suffers from the applied averaging. Additionally, the mean DOPU value for both, the sound enamel and the white spot, decreases for increasing kernel size, as shown in the evaluation (Fig. 4) for the marked regions of interest (ROI, $40 \times 40$ pixels) in Fig. 3. However, the ratio between both values as an achievable contrast increases. Therefore, the visual impression in Fig. 3 is confirmed by the plotted values in Fig. 4.

Following both the visual and the measured results, we decided to use a kernel size of $3 \times 3$ for all subsequent analyses, as it offers a high contrast, which does not considerably increase for larger kernel sizes, and at the same time, maintains a suitable resolution. Moreover, the lower standard deviation (SD) for larger kernel sizes derives from a convergence of ROI and kernel size. It should be noted that for certain cases or questions an asymmetric kernel might be more convenient, as it preserves the resolution in one over the other dimension, and the particular choice should adapt to the particular issue.

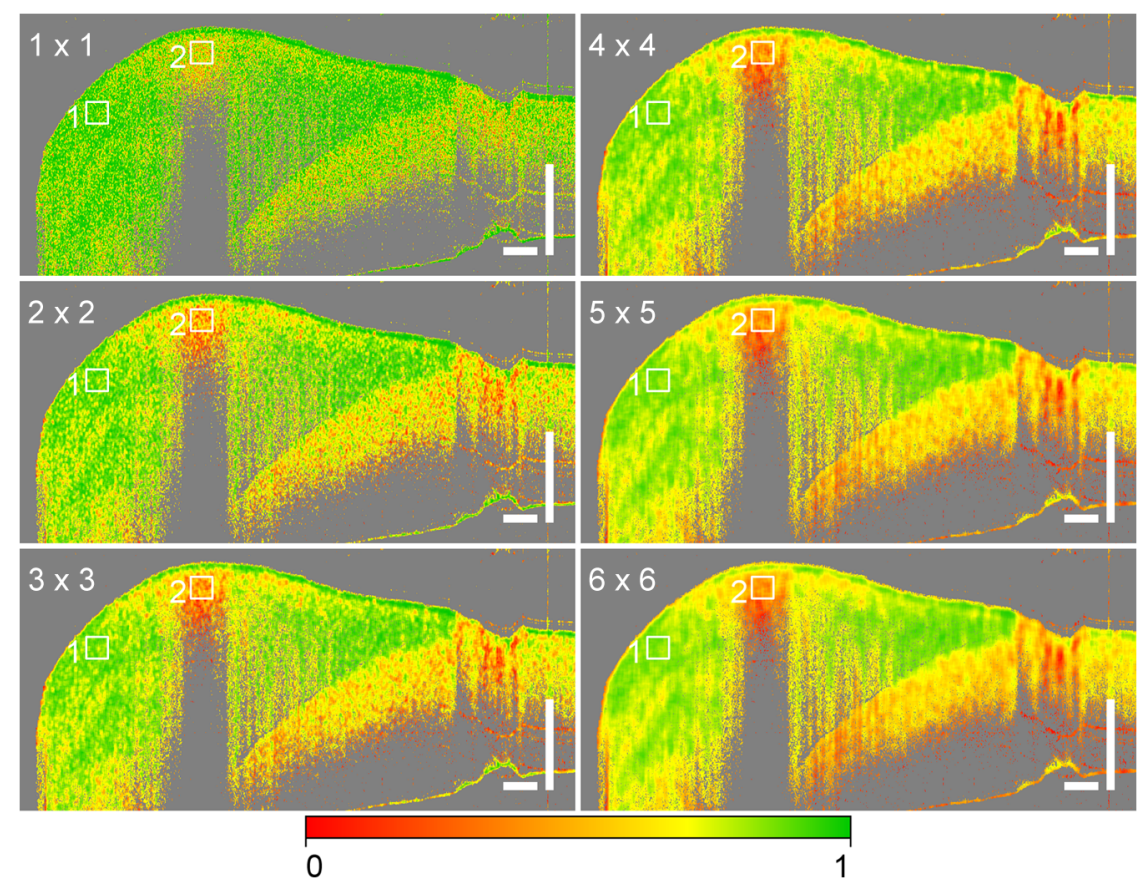

Fig. 3 OCT B-scan with different DOPU evulation kernel sizes as multiples of axial by lateral resolution. Two ROls for sound enamel and white spot are marked. Case $3 \times 3$ equals the DOPU depiction in Fig. 2. Scale bars correspond to $500 \mu \mathrm{m}$. 


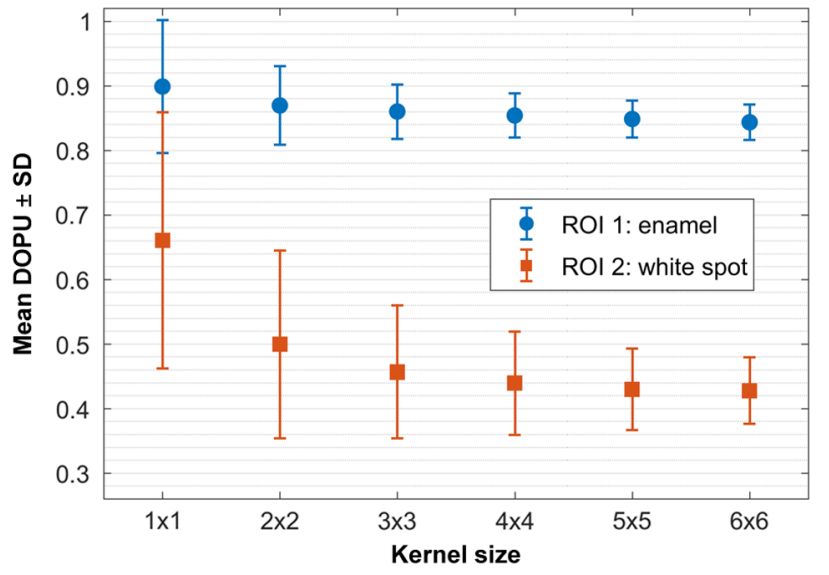

Fig. 4 Mean DOPU values with SD for two different ROI, dependent on the DOPU evulation kernel size. The related ROls are marked in Fig. 3.

\subsection{Imaging Different Caries Stages with DOPU}

To prove the suitability of depolarization imaging with DOPU as an indicative contrast for both early and advanced stages of proximal carious lesions, we selected three exemplary molar teeth for PS-OCT imaging. Teeth 1 and 2 show a noncavitated enamel lesion, which appears as a result of an ongoing demineralization and remineralization process that can finally lead to a collapse of the enamel surface and a cavity (tooth 3 ). As it can be seen in Fig. 5 by means of the presented photographs, the enamel of the white spot has lost its translucency without an additional staining, whereas both the brown spot and the cavity show a considerable discoloration. For all three cases, significant changes of the DOPU appear in those regions that can be identified and assigned as lesions from the photographs. Although it is even possible to visually distinguish areas with differing scattering behavior through the reflectivity images, especially for border areas a clear differentiation seems to be difficult in comparison with the DOPU. Furthermore, the significant DOPU contrast appears to enable a better delimitation of the undermining carious progression, as it happens in case of the cavity. On the other hand, particularly for the direct surface regions, an interpretation of the demineralization progress might depend on the influence of the bright surface reflex, which has to be further investigated. In addition to the enhanced processing algorithms, the application of an index matching substance should be taken into account to solve this issue.

Compilations with all representations, equally shown as in Fig. 2, can be found for all three samples in the appendix.

\section{Summary}

In this study, the application of PS-OCT with circularly polarized incident light on dental hard tissue and depolarization imaging by means of the DOPU for the detection of early carious lesions was demonstrated. Due to the nondestructive and depthresolved imaging abilities, OCT has been a recognized method in the field of dental research for a long time. Similarly, the ability of PS-OCT to examine the birefringent properties of enamel and dentin as well as its pathological alterations has been previously discussed. ${ }^{6,11,15}$ On the other hand, the utilization of the DOPU algorithm in combination with PS-OCT is an established concept in ophthalmologic research. ${ }^{8,16,18}$ To the best of our knowledge, this is the first time that the DOPU algorithm is applied for the detection of carious lesions in dentistry. We consider it as a promising method for the identification and delimitation of early carious lesions in particular but also the assessment of the severity of an undermining caries and a cavity.

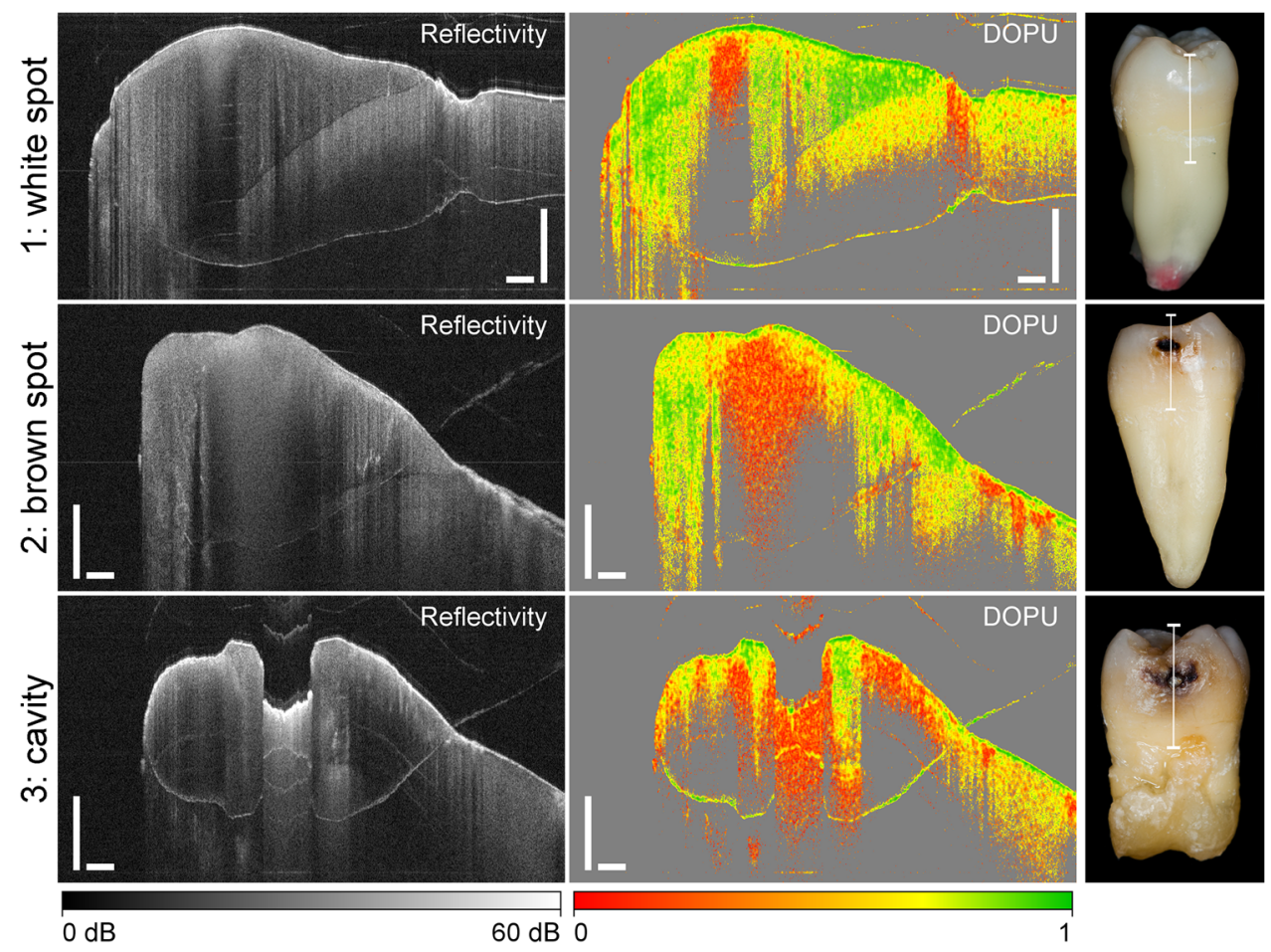

Fig. 5 Comparison of reflectivity and DOPU for B-scans of three molar teeth with different proximal lesions: white spot, brown spot, and cavity. Photographs of the examined teeth with a marking of the corresponding B-scan region are presented. Scale bars correspond to $500 \mu \mathrm{m}$. 
For this purpose, we compared the B-scans of intensities measured by both the co- and cross-polarization channels as well as the resulting reflectivity with retardation, optical axis orientation, and DOPU images of three molar teeth with proximal carious lesions. The findings demonstrate that DOPU seems to be the most appropriate representation to detect carious lesions and its extension for both early and advanced stages. Furthermore, we compared different sizes of the DOPU evaluation kernel and found that with respect to the axial and lateral resolutions a window of $3 \times 3$ is most suitable in this case. For the future, PS-OCT and depolarization imaging of dental demineralization with the aid of the DOPU algorithm could support a deeper understanding of caries progression in vitro. Although it has not yet been tested in vivo, the application of this technique with endoscopic fiber optics could consequently enable an early detection of carious lesions noninvasively. Due to the limited penetration depth of the OCT signal, proximal contact surfaces might be more challenging than near-gingival spaces that could be reached with suitable miniaturized optics, whereas occlusal, buccal, and lingual lesions should be well accessible with an endoscope. Since DOPU images provided a superior contrast, DOPU could be an appropriate PS-OCT image representation when utilizing OCT as a diagnostic screening tool.

\section{Appendix}

To provide a complete comparison of the considered image representations, co- and cross-polarization intensities, retardation, and fast axis orientation are displayed in Figs. 6-8 in addition to the reflectivity and DOPU images, which are already shown in Fig. 5 for white spot, brown spot, and cavity.

\section{Disclosures}

The authors declare that there are no conflicts of interest related to this article.
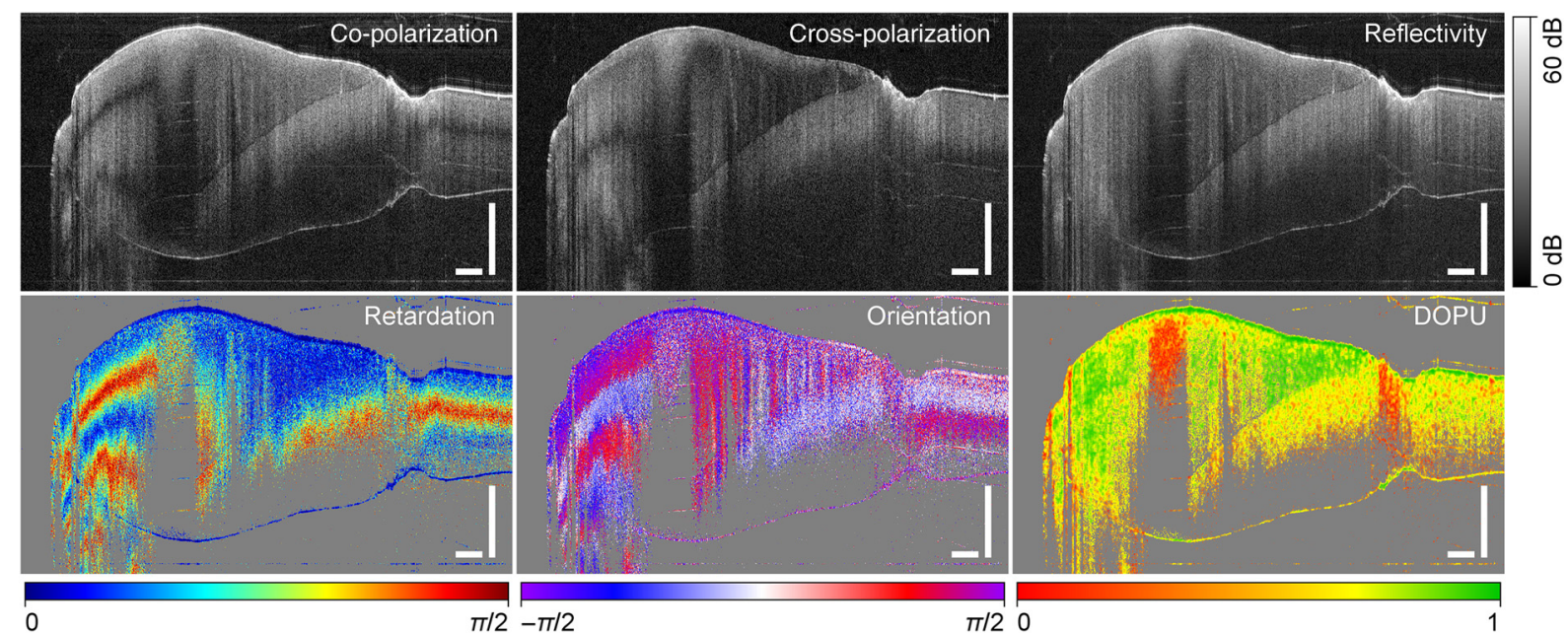

0

$\pi / 2-\pi / 2$

$\pi / 20$

Fig. 6 Co- and cross-polarization channels, calculated reflectivity, retardation, fast axis orientation, and DOPU $(3 \times 3$ kernel) of the B-scan shown in Fig. 5 for "white spot" are displayed. Scale bars correspond to $500 \mu \mathrm{m}$.
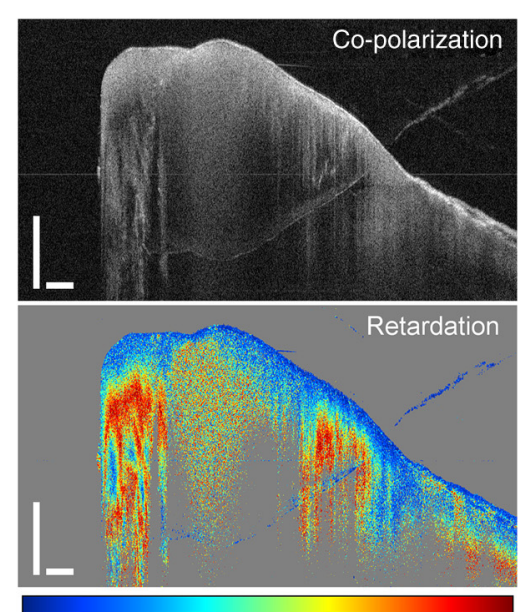

0
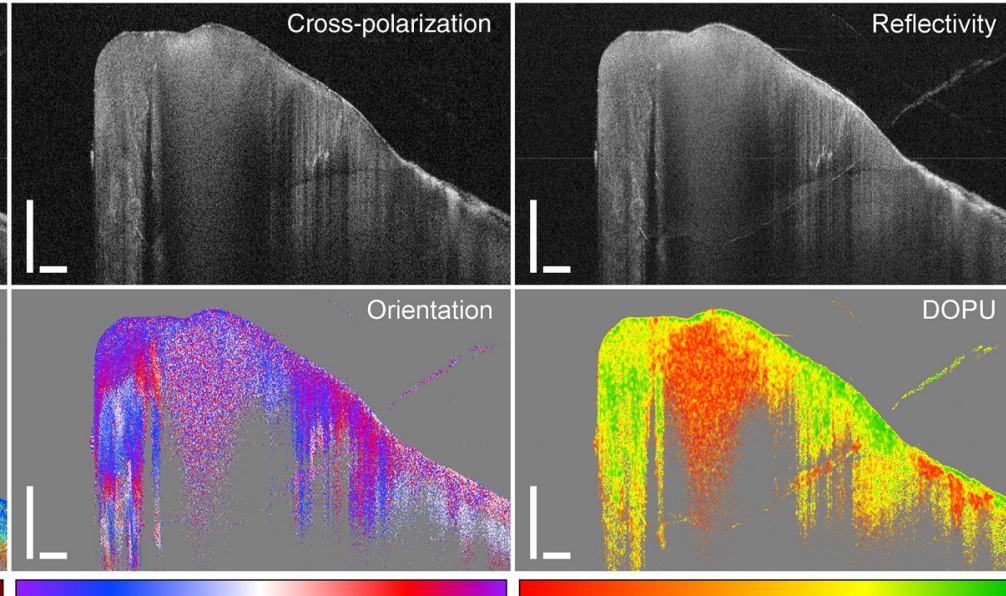

DOPU

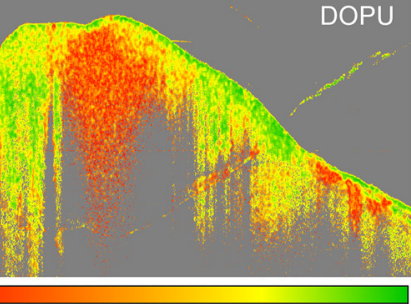

$-\pi / 2$

$\pi / 20$

Fig. 7 Co- and cross-polarization channels, calculated reflectivity, retardation, fast axis orientation, and DOPU $(3 \times 3$ kernel) of the B-scan shown in Fig. 5 for "brown spot" are displayed. Scale bars correspond to $500 \mu \mathrm{m}$. 


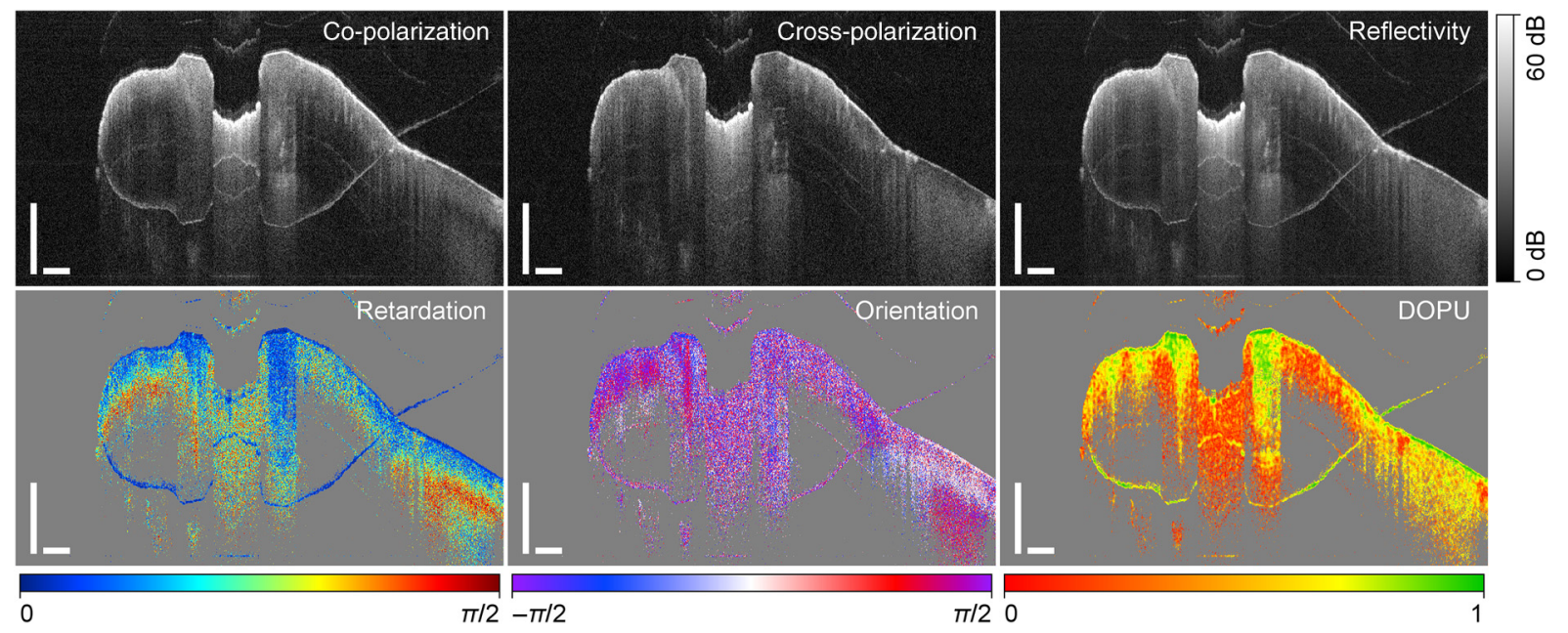

Fig. 8 Co- and cross-polarization channels, calculated reflectivity, retardation, fast axis orientation, and DOPU $(3 \times 3$ kernel) of the B-scan shown in Fig. 5 for "cavity" are displayed. Scale bars correspond to $500 \mu \mathrm{m}$.

\section{Acknowledgments}

This project was supported by the European Union/European Social Fund (ESF) and the Free State of Saxony within the ESF junior research group "Optical Technologies in Medicine" (Project No. 100270108). The author Jonas Golde was supported by the European Union/ESF and the Free State of Saxony within a doctoral scholarship (Project No. 100284305).

\section{References}

1. I. A. Pretty, "Caries detection and diagnosis: novel technologies," J. Dent. 34(10), 727-739 (2006).

2. F. Brouwer et al., "Detecting secondary caries lesions: a systematic review and meta-analysis," J. Dent. Res. 95(2), 143-151 (2016).

3. B. W. Colston et al., "Dental OCT," Opt. Express 3, 230-238 (1998).

4. A. Baumgartner et al., "Optical coherence tomography of dental structures," Proc. SPIE 3248, 130-137 (1998).

5. D. Fried et al., "Nature of light scattering in dental enamel and dentin at visible and near-infrared wavelengths," Appl. Opt. 34(7), 1278-1285 (1995).

6. H. Schneider et al., "Dental applications of optical coherence tomography (OCT) in cariology," Appl. Sci. 7(5), 472 (2017).

7. J. Li et al., "Speckle reduction and lesion segmentation of OCT tooth images for early caries detection," in Annual Int. Conf. of the IEEE Engineering in Medicine and Biology Society, pp. 11491452 (2009).

8. B. Baumann, "Polarization sensitive optical coherence tomography: a review of technology and applications," Appl. Sci. 7(5), 474 (2017).

9. J. F. de Boer et al., "Polarization sensitive optical coherence tomography-a review," Biomed. Opt. Express 8(3), 1838-1873 (2017).

10. X.-J. Wang et al., "Characterization of dentin and enamel by use of optical coherence tomography," Appl. Opt. 38(10), 2092-2096 (1999).

11. M. Everett et al., "Non-invasive diagnosis of early caries with polarization sensitive optical coherence tomography (PS-OCT)," Proc. SPIE 3593, 177-182 (1999).

12. P. Ngaotheppitak et al., "Measurement of the severity of natural smooth surface (interproximal) caries lesions with polarization sensitive optical coherence tomography," Laser Surg. Med. 37(1), 78-88 (2005).

13. R. S. Jones et al., "Remineralization of in vitro dental caries assessed with polarization-sensitive optical coherence tomography," J. Biomed. Opt. 11(1), 014016 (2006).

14. J. C. Simon et al., "Near-IR and CP-OCT imaging of suspected occlusal caries lesions," Laser Surg. Med. 49(3), 215-224 (2017).
15. D. Fried et al., "Imaging caries lesions and lesion progression with polarization sensitive optical coherence tomography," J. Biomed. Opt. 7(4), 618-627 (2002).

16. E. Götzinger et al., "Retinal pigment epithelium segmentation by polarization sensitive optical coherence tomography," Opt. Express 16(21), 16410-16422 (2008).

17. N. Lippok et al., "Degree of polarization (uniformity) and depolarization index: unambiguous depolarization contrast for optical coherence tomography," Opt. Lett. 40(17), 3954-3957 (2015).

18. S. Makita et al., "Degree of polarization uniformity with high noise immunity using polarization-sensitive optical coherence tomography," Opt. Lett. 39(24), 6783-6786 (2014).

19. N. Ortega-Quijano et al., "Enhanced depolarization contrast in polarization-sensitive optical coherence tomography," Opt. Lett. 41(10), 2350-2353 (2016).

20. A. Miyazawa et al., "Generation and optimization of superpixels as image processing kernels for Jones matrix optical coherence tomography," Biomed. Opt. Express 8(10), 4396-4418 (2017).

21. M. R. Hee et al., "Polarization-sensitive low-coherence reflectometer for birefringence characterization and ranging," J. Opt. Soc. Am. B 9(6), 903-908 (1992).

22. J. Schindelin et al., "Fiji: an open-source platform for biological-image analysis," Nat. Methods 9(7), 676-682 (2012).

23. J. M. Schmitt et al., "Speckle in optical coherence tomography," J. Biomed. Opt. 4(1), 95-105 (1999).

24. Z. Meng et al., "Measurement of the refractive index of human teeth by optical coherence tomography," J. Biomed. Opt. 14(3), 034010 (2009).

25. Central German Ethics Committee, "The use of human body materials for the purposes of medical research" Dtsch. Arztebl. 100(23), A1632 (2003).

26. J. K. Avery, P. F. Steele, and N. Avery, Oral Development and Histology, 3rd ed., Thieme, Georg Thieme Verlag, Stuttgart, Germany (2002).

27. L. Raue et al., "New insights in prism orientation within human enamel," Arch. Oral Biol. 57(3), 271-276 (2012).

28. N. Lippok et al., "Single input state, single-mode fiber-based polarization-sensitive optical frequency domain imaging by eigen polarization referencing," Opt. Lett. 40(9), 2025-2028 (2015).

29. Y. Chen et al., "Polarization memory effect in optical coherence tomography and dental imaging application," J. Biomed. Opt. 16(8), 086005 (2011).

Jonas Golde is a PhD student at the Technische Universität (TU) Dresden. He received his MSc degree in physics from the TU Dresden in 2016 with a thesis on polarization sensitive optical coherence tomography (PS-OCT). Since then, he has been focused on the development and application of PS-OCT systems in the fields of dentistry and otolaryngology. His research interests include the 
combination of PS-OCT with complementary optical technologies, such as multiphoton microscopy and hyperspectral imaging.

Florian Tetschke received his MSc degree in biomedical engineering from the University of Applied Sciences Jena in 2013. He contributed to research projects at the Biomagnetic Center of the University Hospital in Jena, at the Polytechnical University of Catalonia, and the Institute of Biomedical Engineering at the TU Dresden. Currently, he is a research associate at the junior research group, "Optical Technologies in Medicine" at the TU Dresden. His research interest focuses on the development and application of optical technologies, such as hyperspectral imaging, optical coherence tomography (OCT), and Raman spectroscopy.

Julia Walther is a senior researcher at the TU Dresden. She received her diploma in physics technology and biomedical engineering from the University of Applied Sciences Mittweida in 2006 and her PhD in medical physics and biomedical optics from the TU Dresden in 2010. Her current research interests include the advancement of OCT for different applications in dentistry and otolaryngology as well as the analysis and extension of diverse theoretical models for Doppler OCT (DOCT).

Tobias Rosenauer is a dentist and a research associate at the University Clinic Carl Gustav Carus, Dresden. He graduated in 2016 and has since worked in the Department of Operative Dentistry, TU Dresden. $\mathrm{He}$ is currently writing his doctoral thesis and his current research concerns cariological aspects as well as optical imaging.

Franz Hempel received his BSc degree in physics from the TU Dresden in 2017. He wrote his thesis on PS-OCT in the work group
Clinical Sensoring and Monitoring, the Medical Faculty, TU Dresden. His research interests include the application of PS-OCT in biomedical imaging.

Christian Hannig is head of the Department of Operative Dentistry, TU Dresden. Furthermore, he is the dean for dental education. He has been a dentist since 2000 and has worked at Göttingen and Freiburg University before coming to Dresden. His main field of research is bioadhesion in the oral cavity. He is the author of more than 100 international and national journal papers. His research has been funded several times by the German Research Foundation.

Edmund Koch received his diploma and $\mathrm{PhD}$ in physics from the University of Marburg. He worked as a senior researcher at the Drägerwerke AG, Lübeck, and thereafter, he became the professor for laser and optics at the Lübeck University of Applied Sciences. $\mathrm{He}$ is currently the head of the work group Clinical Sensoring and Monitoring, the Faculty of Medicine, the TU Dresden. His main research interests are OCT and spectroscopic imaging.

Lars Kirsten is a senior researcher at the research group Clinical Sensoring and Monitoring of Professor Dr. Edmund Koch at the TU Dresden. He joined the research group in 2009 and received his diploma in physics in 2010 and his $\mathrm{PhD}$ (Dr.rer.nat.) in 2015. Since 2016, he is the leader of the junior research group Optical Technologies in Medicine. His current research interests include OCT, especially the further development of PS-OCT, DOCT, endoscopic OCT, and their applications in otolaryngology, dentistry, and biomedical basic research. 